\title{
A VLBI and MERLIN Survey of faint, compact radio sources
}

\author{
S.T. Garrington ${ }^{a}$ M.A. Garrett ${ }^{b}$ A. Polatidis ${ }^{b, c}$ \\ ${ }^{a}$ Nuffield Radio Astronomy Laboratories, The University of Manchester, Jodrell \\ Bank, Macclesfield SK11 9DL, UK \\ ${ }^{\mathrm{b}}$ Joint Institute for VLBI in Europe, Postbus 2, 7990 AA Dwingeloo, The \\ Netherlands \\ ${ }^{\mathrm{c}}$ Onsala Space Observatory, Onsala, Sweden
}

\begin{abstract}
We present preliminary results from a VLBI survey at $\lambda=6 \mathrm{~cm}$ of a sample of 35 sources with flux densities of $2-100 \mathrm{mJy}$. These sources were selected from the VLA FIRST survey at $\lambda=20 \mathrm{~cm}$, in a 3 degree field around the bright calibrator $1156+295$, simply by imposing $S_{20}>10$ mJy and $\theta<5$ arcsec. MERLIN observations at $\lambda 6 \mathrm{~cm}$ detected $70 / 127$ of these sources with a threshold of $2 \mathrm{mJy}$ at 50 mas resolution and the closest 35 of these to the calibrator were observed with the VLBA $+E V N$ in snapshot mode at $\lambda 6 \mathrm{~cm}$. These sources are a mixture of flat and steep-spectrum sources and include: weak flat-spectrum nuclei of large radio galaxies, low power AGN in nearby galaxies and radio quiet quasars. With these short observations, the sensitivity is limited and most appear as either core-jets or simple point sources on the milliarcsec scale. Nonetheless, it is encouraging that with only 10 minutes observation per source, at least $35 \%$ of all sources with $S_{20}>10$ mJy can be detected and imaged with global $6 \mathrm{~cm}$ VLBI.
\end{abstract}

Key words: radio continuum:, galaxies: nuclei PACS: 98.62.Js, 98.62.-g

\section{Introduction}

The new VLA surveys, FIRST (Becker et al., 1995) and NVSS (Condon et al, 1998 ), have opened up the mJy radio population on a vast scale, but even the higher resolution FIRST survey does not resolve the majority of sources which it detects. The tendency for the fainter sources to be considerably smaller has been known for some years (Oort, 1987) and is strikingly demonstrated by 
recently compiled statistics of the large MIT VLA surveys, where $47 \%$ of the sources brighter than 50 mJy imaged with 0.25 arcsec resolution at $8.4 \mathrm{GHz}$ were found to be unresolved (Fletcher et al., 1998)

However, this mJy population has yet to be systematically investigated with VLBI, previous VLBI surveys have so far been limited to flux densities > 100 mJy (Polatidis et al., 1995; Beasley et al., 1996) and often these samples have been restricted to relatively flat spectrum sources $(\alpha<0.5)$. High resolution images will be of interest for population studies of weak AGN, such as the evolutionary schemes recently proposed by Wall and Jackson (1997), and will be a valuable input to statistical studies of gravitational lensing, since the compact mJy radio source population is the 'parent population' of the lensed sources being detected in the CLASS and MIT surveys (Falco et al., 1998).

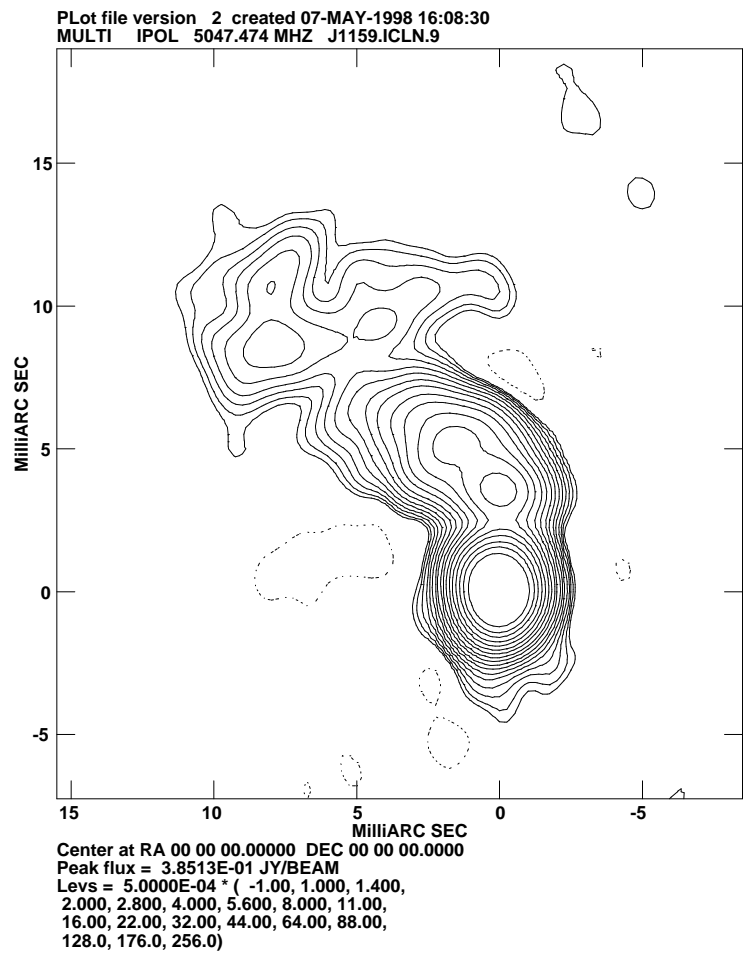

Fig. 1. Global $6 \mathrm{~cm}$ VLBI image of $1156+295$, used as the phase calibrator.

VLBI observations of mJy sources are only possible by using the technique of phase referencing. The density of the new VLA surveys is such that we are now free to choose an ideal phase calibrator source and then simply select targets from the surrounding field.

The initial swathe of the FIRST survey included the area around $1156+295$, an interesting source in its own right (Figure 1, see also Hong, these proceedings) but also an ideal phase calibrator. We therefore defined a $3 \times 4$ degree field centred on this source and simply selected as potential targets all 127 sources from the FIRST catalogue in this area with $S_{1.4}>10 \mathrm{mJy}$ and LAS $<5.0$ 
arcsec.

\section{MERLIN Observations}

In order to select those sources with sufficient emission on milliarcsec scales for VLBI imaging, we used MERLIN at $\lambda 6 \mathrm{~cm}$, observing each source for $3 \times 5$ minutes, giving a detection threshold of about $2 \mathrm{mJy}$ with 50 mas resolution (Garrett \& Garrington, 1998). This is a savage cut for steep-spectrum sources, which would have to be very compact to be detected by MERLIN at this level, but we would expect to detect the majority of flat-spectrum sources, which should account for $<30 \%$ of the sample. Perhaps surprisingly, just over half of the list, 70 sources, were detected. These appeared to be mostly point-sources, but there were a few simple double sources, and several marginal detections of complex emission from the lobes of large double sources, which, given the very simple selection criteria, are not excluded from the target list.

VLA A-configuration observations at 1.4 and $8.4 \mathrm{GHz}(1.0$ and 0.25 arcsec resolutions) were made of the final target list in order to image structure on intermediate scales and to determine spectral indices. MERLIN + EVN observations at $18 \mathrm{~cm}$ have also been made and and the EVN data are now being correlated.

\section{Global VLBI Observations at $\lambda=6 \mathrm{~cm}$}

Global $\lambda 6 \mathrm{~cm}$ VLBI observations (18 telescopes; VLBA + EVN) were made earlier this year. To meet scheduling constraints, 35 sources closest to the phase calibrator and detected by MERLIN at $6 \mathrm{~cm}$ were selected. With $6 \times 2$ minutes observation per source, we achieved 2 mas resolution and a detection threshold of 1 - 2 mJy. The source positions were known to within a few mas from the MERLIN observations, reducing the risk of false detections.

Only 8 of the 35 sources were not detected in these global VLBI observations. These were bright, steep-spectrum and clearly extended on intermediate scales three were in fact lobes of large double sources, four were CSS sources with sizes > 200 mas and one source was a marginal detection with MERLIN. Indeed, we would not have expected to have detected these sources, but they were not excluded by the 'blind' selection process.

Of the 27 sources which were detected, there were roughly equal numbers of flat and steep-spectrum sources. 

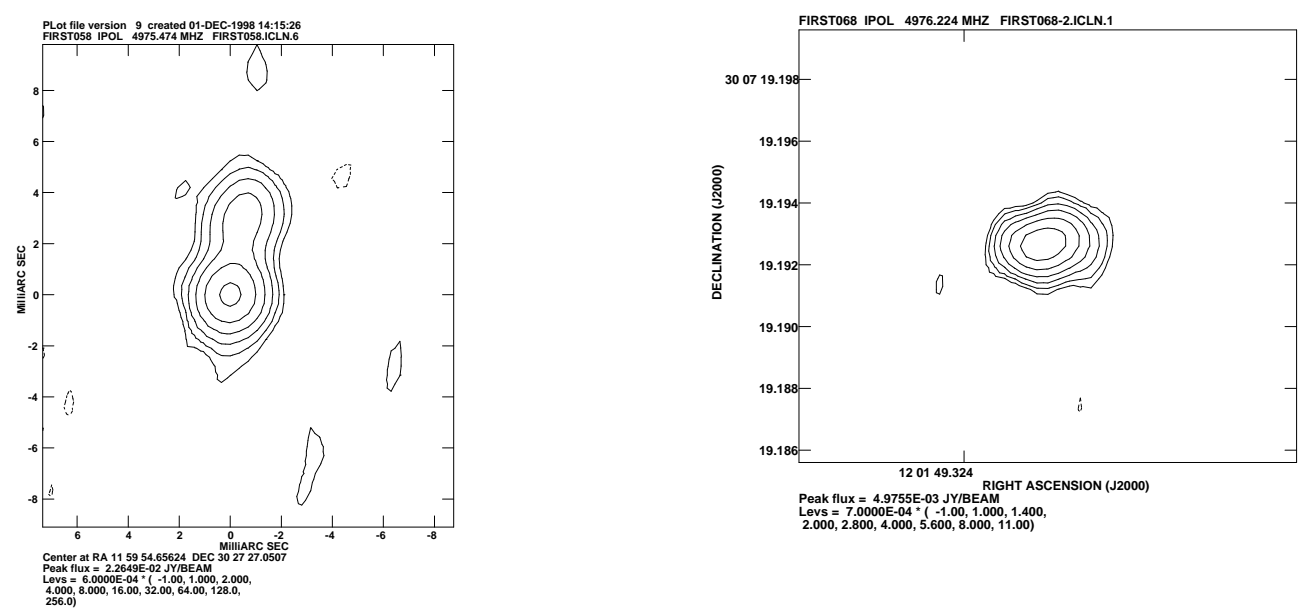

Fig. 2. These faint milliarcsec flat spectrum sources are associated with bright $(\mathrm{R}=14$ and 16) galaxies. Neither have significant extended radio emission on scales larger than this.

The brighter flat-spectrum sources are associated with nearby red galaxies. Two are the nuclei of arcminute scale radio galaxies, but otherwise they have no extended emission on intermediate scales (0.1 - 10 arcsec), and two are quasars. On the milliarcsecond scale, these sources mostly have core-jet structures. The fainter sources $(10-50 \mathrm{mJy})$ have no optical identifications on the POSS and appear to be unresolved from mas to arcsecond scales. These flat-spectrum sources have $T_{\mathrm{b}} \sim 10^{10}-10^{11} \mathrm{~K}$.
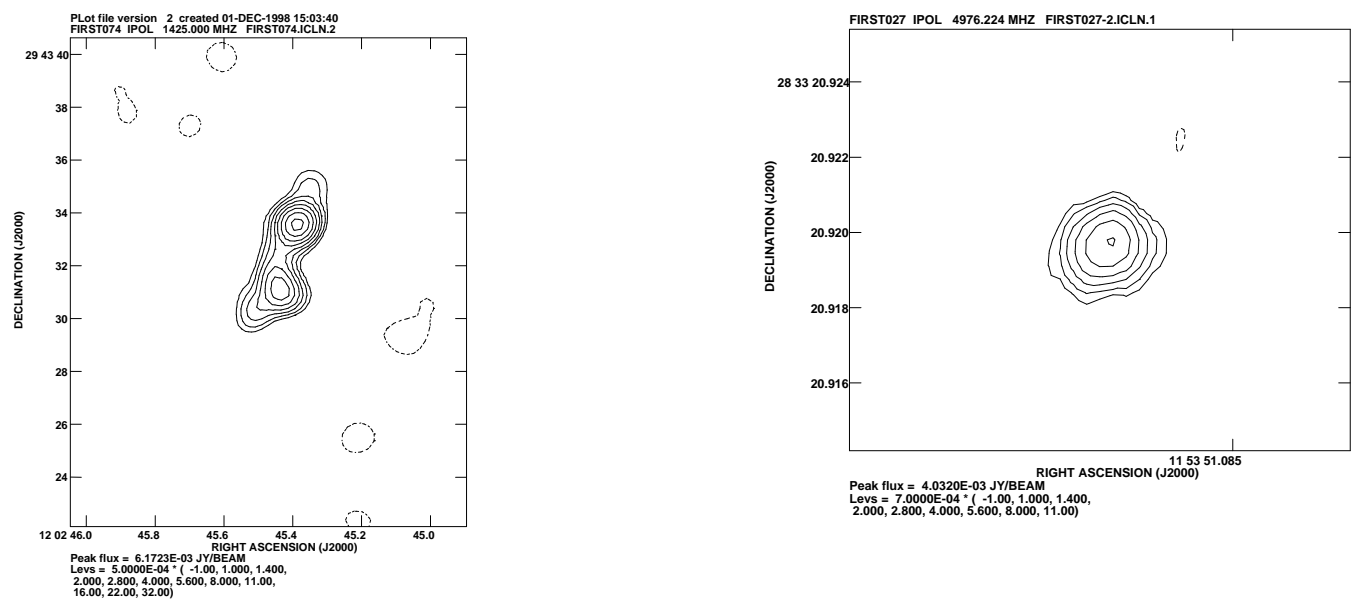

Fig. 3. The VLA A-configuration $20 \mathrm{~cm}$ map on the left shows a flat spectrum core and an extended 5 arcsec jet to the south in this 18 mJy source. There may also be lower surface brightness features resolved out by this image with a total flux density of a few mJy. The $6 \mathrm{~cm}$ global VLBI phase referenced image on the right, shows the $5 \mathrm{mJy}$ core which is barely resolved. The slight extension may be due to residual phase errors and a realistic upper limit on the component size is 1 mas, confirmed using the VLBA data alone. This source may be identified with one of a group of faint galaxies. 
Of the steep-spectrum sources, six are barely resolved: these may be faint GPS sources with spectral peaks below $1 \mathrm{GHz}$ and expected sizes of 1 - 10 mas (Snellen, these proceedings). If so, this would indicate quite a high fraction of GPS sources at this flux level. The sources with intermediate spectral indices have, as expected, arcsecond scale jets and mas cores. The largest example is a 5 arcsecond triple source, which just fell within the initial selection.

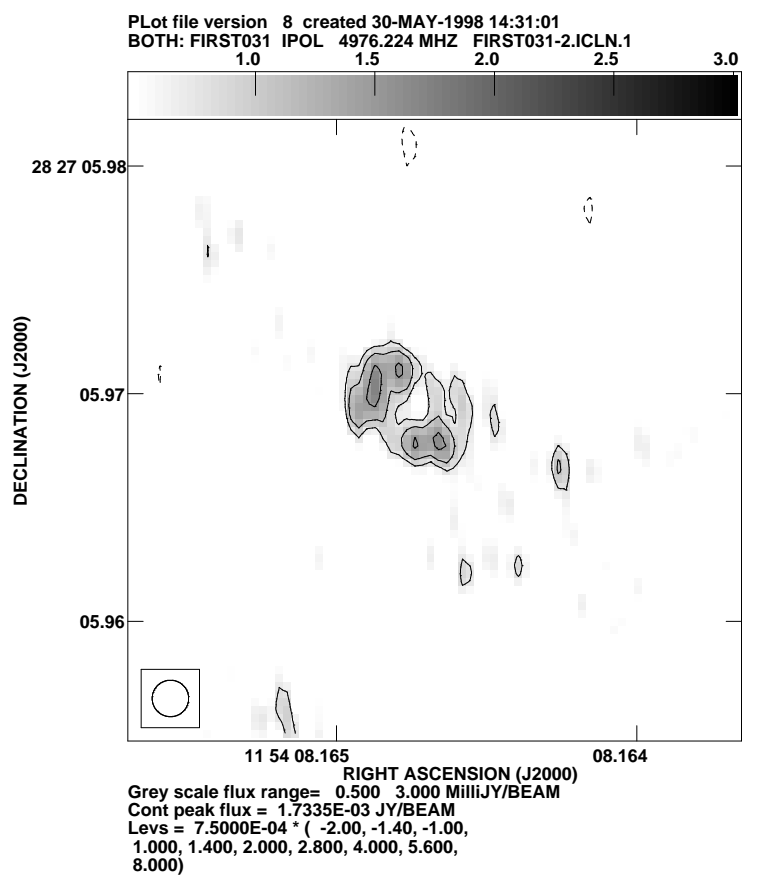

Fig. 4. This steep spectrum source has no optical identification on the POSS plate and no significant extended emission on arcsec scales. The phase-referenced image shows a complex, ring-like structure.

One source has a rather unusual 5 mas diameter ring-like structure. Optically, this is an empty field down to 21 mag. The nearest optical objects are 30 arcsec away and are faint. The source has a spectral index of 0.80 with no extended radio structure on any scales from 50 mas to several arcminutes, except perhaps a few mJy within 0.5 arcsec of the 'core' and its nearest radio neighbour is a 4 mJy source 2 arcminutes away. Ascribing this distorted structure to gravitational lensing would imply a lens mass of $\sim 10^{6}$ solar masses. The expected probability for lensing on this scale, even assuming all normal galaxies harbour massive black holes is tiny $\left(3 \times 10^{-9}\right)$. Alternatively, this may be an extremely distorted jet, perhaps like 3C119 (Nan Ren-dong et al. 1991) or Mkn 501 Conway \& Wrobel, 1995) but 50 times fainter and several times smaller. More sensitive VLBI observations are required to investigate this source further. 


\section{Conclusions}

We have produced VLBI images of an un-biased sample (no spectral index or optical selection) of faint, compact radio sources. With only 10 minutes observation per source, approximately $35 \%$ of all sources with $S_{20}>10 \mathrm{mJy}$ can be detected on global VLBI baselines at $6 \mathrm{~cm}$.

This project has used the simplest of selection criteria and we have seen that that at the mJy level, the VLBI sky shows a broad range of flat and steep spectrum sources, apparently distant and nearby. In order to complete the statistical picture on this small sample, we are awaiting optical imaging and spectroscopic observations as well as the EVN 18cm observations to complete radio picture. Perhaps the most important lesson from this pilot VLBI survey of faint radio sources is that the mJy population is readily accessible with current VLBI techniques. The new VLA surveys provide a huge resource for this type of work and MERLIN can be used as a very efficient filter for selecting potential VLBI targets.

\section{References}

Beasley, A., Dhawan, V., Fomalont et al., in: Ekers, R., Fanti, C., Padrielli, L. (eds) Extragalactic Radio Sources - Proceedings of IAU Symposium 175, Kuwer, Dordrecht, p. 527.

Becker, R. et al. 1995, ApJ., 450, 559.

Condon, J., Cotton, W., Greisen, E., Yin, Q., Perley, R.Taylor, G. \& Broderickk, J., 1998, AJ, 115, 1693

Conway, J. \& Wrobel, J. ApJ 439,98.

Falco, E., Kochanek, C. \& Munoz, J., 1998, ApJ, 494, 47

Fletcher, A., Burke, B., Conner, S. Lehar, J., \& Herold, L., 1998, in: Bremer, M., Jackson, N., \& Perez-Fournon, I. (eds) Observational Cosmology with the New Radio Surveys, Kluwer, Dordrecht, p. 255.

Garrett, M.A. \& Garrington, S.T., 1998, in: Zensus, J.A., Taylor, G.B., \& Wrobel, J.M. (eds), IAU Colloquium 164, ASP Conference Series, 144, p 145.

Oort, M., 1988, A\&A, 193, 50.

Polatidis, A., Wilkinson, P., Xu, W., Readhead, A., Pearson, T., Taylor, G. \& Vermeulen, R., 1995, ApJS, 98, 1.

Nan Ren-Dong, Schilizzi, R., van Breugel, W., Fanti, C., Fanti, R., Muxlow, T., \& Spencer, R., 1991, A\&A 245, 449

Wall, J. \& Jackson, C., 1997, MNRAS, 290, 17 